\title{
Low-noise properties of squeezed light in transmission chains formed from nonlinear alternating attenuators and amplifiers
}

\author{
P. García-Fernández and F. J. Bermejo \\ Instituto de Estructura de la Materia, Consejo Superior de Investigaciones Científicas, Serrano 119, 28006 Madrid, Spain \\ (Received 10 August 1987; revised manuscript received 1 December 1987)
}

\begin{abstract}
An idealized model of an optical communication link in which identical sections of nonlinear attenuating fiber alternate with identical amplifiers is considered. Signal-to-noise ratios of chains of these elements for input squeezed and coherent light have been numerically evaluated. It is found that input squeezed light and nonlinear attenuators improve the signal-to-noise ratios with respect to coherent inputs and linear chains. In the case of three-photon attenuators, the noise and signalto-noise ratio remain practically constant from certain elements of the chain. Moreover, the influence of the attenuation coefficient and the input mean photon number on the signal-to-noise ratios was considered, as well as chains with nonlinear amplifiers and chains in which the amplifier gain does not exactly compensate for the fiber attenuation.
\end{abstract}

A long optical transmission line is usually broken up into shorter sections separated by electrical regenerators to maintain the signal at a sufficiently high level. The noise properties of an all-optical system in which linear optical amplifiers alternate with attenuating fiber sections along a long transmission line have previously been considered. ${ }^{1,2}$ On the other hand, it has been shown that the two-photon coherent states (squeezed states) have a significant potential for improving free-space optical communications. ${ }^{3}$ Moreover, the influence of linear optical amplifiers on the low-noise properties of squeezed light have been determined by application to a model for a long transmission link formed from alternating attenuators and amplifiers. ${ }^{4}$

The recent success in generating and detecting squeezed light ${ }^{5}$ brings closer the possibility of using such light in various applications that could take advantage of its low-noise properties. In this Brief Report we study the low-noise properties of squeezed light in an idealized model of a transmission link formed from alternating nonlinear attenuators and optical amplifiers.

The elements of the chain have been considered within a model that consists of a single-mode radiation field of frequency $\omega$, which interacts with a large number of identical two-level atoms confined to a volume $V$ of an optical cavity via $n$-photon transitions in a Markovian-like process. The irreversible dynamics of the statistical properties of the field is known to be governed by the following master equation for the density operator $\rho$ of the field in the interaction picture: ${ }^{6,7}$

$$
\begin{aligned}
\frac{\partial \rho}{\partial t}= & -\beta^{(n)} N_{1}\left(a^{\dagger n} a^{n} \rho-2 a^{n} \rho a^{+n}+\rho a^{+n} a^{n}\right) \\
& -\beta^{(n)} N_{2}\left(a^{n} a^{\dagger n} \rho-2 a^{\dagger n} \rho a^{n}+\rho a^{n} a^{\dagger n}\right),
\end{aligned}
$$

where $a$ and $a^{\dagger}$ are the usual annihilation and creation operators for the photon field, respectively, $N_{1}, N_{2}$ are the average populations of the active levels, and $\beta^{(n)}$ is a coupling constant proportional to the square of the atom- ic nonlinear transition matrix element, with dimension (time $^{-1}$. The solution of Eq. (1) as well as the relevant computational details are given in Refs. 8-14.

The ideal squeezed state ${ }^{8,4}|\alpha ; s, \theta\rangle$ has uncertainty variances that take minimum and maximum values

$$
\left\langle\left(\Delta a_{1}^{\prime}\right)^{2}\right\rangle=\frac{1}{4} \exp (-2 s)
$$

and

$$
\left\langle\left(\Delta a_{2}^{\prime}\right)^{2}\right\rangle=\frac{1}{4} \exp (2 s)
$$

parallel to a pair of field axes inclined at angles $\theta / 2$ to the axes defined by the two quadrature components of the field $a_{1}=\left(a+a^{\dagger}\right) / 2, a_{2}=i\left(a^{\dagger}-a\right) / 2$. The squeeze parameter $s$ is real and positive, and the angle $\theta$ runs from 0 to $2 \pi$. The nonclassical regime corresponds to field variances smaller than $\frac{1}{4}$. The expectation values of the field quadratures for the ideal squeezed state are given by

$$
\begin{aligned}
& \left\langle a_{1}\right\rangle=|\alpha| \cos (\arg \alpha), \\
& \left\langle a_{2}\right\rangle=|\alpha| \sin (\arg \alpha),
\end{aligned}
$$

and the signal-to-noise ratio $\left(R_{\mathrm{SN}}\right)$

$$
R_{\mathrm{SN}}=\frac{\left\langle a_{1}\right\rangle^{2}}{\left\langle\left(\Delta a_{1}\right)^{2}\right\rangle},
$$

where $a_{1}$ is the quadrature component with reduced fluctuations of the squeezed input. It has been shown ${ }^{4}$ that the maximum signal-to-noise ratio is obtained for $\arg \alpha=\theta / 2$ and is given by

$$
R_{\mathrm{SN}}^{\max }=4|\alpha|^{2} \exp (2 s) \text {. }
$$

When $s=0$ we recover $R_{\mathrm{SN}}^{\max }$ for a coherent input $|\alpha\rangle$.

Amplifiers that take no account of the phase of the input light are typified by the inverted atomic population. ${ }^{4}$ For this amplifier model, the input and output field expectation values are related by

$$
\left\langle a_{1}\right\rangle=G^{1 / 2}\left\langle a_{1}\right\rangle_{0},
$$

where $G$ is a constant determined by the properties of the 
atomic transition, the atomic density and the length of the amplifying region. The model can act as an attenuator as well as an amplifier, and the corresponding ranges of parameter values are for the attenuator,

$$
G<1, \quad 0 \leq P \leq \frac{1}{2},
$$

and for the amplifier,

$$
G>1, \quad \frac{1}{2} \leq P \leq 1,
$$

where $P=N_{2} /\left(N_{1}+N_{2}\right)$, and $N_{1}, N_{2}$ are the numbers of atoms in the upper and lower levels of the transition. It has been shown ${ }^{4}$ that the amplifier minimum noise is attained for $P=1$ and it is the case we have dealt with.

The effects of the fiber attenuation are formally the same as those of the phase-insensitive amplifier but with the gain $G$ replaced by the loss $K$ and the atoms all taken to be in their ground states $(P=0)$. The required connections between the fiber-input and -output mean field and photon number are thus ${ }^{4}$

$$
\begin{aligned}
& \left\langle a_{1}\right\rangle=K^{1 / 2}\left\langle a_{1}\right\rangle_{0}, \\
& \langle n\rangle=K\langle n\rangle_{0} .
\end{aligned}
$$

Finally, it is convenient to choose an amplifier gain that exactly compensates for the fiber loss, $K G=1$, so that the mean-output field $\left\langle a_{1}\right\rangle$ equals the mean-input signal field $\left\langle a_{1}\right\rangle_{0}$. On the other hand, the phase angles were assumed so that the input has the maximum signalto-noise ratio $(\arg \alpha=\theta / 2)$. In this article we numerically study the variation of the signal, the noise and the signal-to-noise ratio along a chain of alternating attenuators and amplifiers, both in the linear and nonlinear cases, for squeezed and coherent inputs. It should be noted that we deal with an idealized model that does not take into account the deleterious effects due to nonlinearities.

The case where the elements of the chain are linear attenuators and linear amplifiers under the conditions of minimum noise is considered first. Figure 1 shows the evolution of the noise and the signal-to-noise ratio along the chain for input squeezed and coherent states. We will represent the decays over the attenuation sections by straight lines for simplicity, which are not intended to show the detailed behaviors as functions of distance along the fiber.

Figure 1(a) shows the behavior of the noise for an input squeezed state with squeezing parameter $s=1$, squeezing phase $\theta=20^{\circ}$, phase of the complex amplitude $\arg \alpha=10^{\circ}$, and input mean photon number $\bar{n}_{0}=20$. The attenuation coefficient is $K=0.82$, and the elements of the chain (labeled by $m$ ) satisfy the relation $K G=1$. The evolution of the noise for the coherent input is also given. It can be seen that the noise corresponding to the squeezed input remains below that of the coherent input. The noise can be reduced with an input squeezing phase $\theta=0$, since the squeezing phase decreases the initial degree of squeezing. On the other hand, the attenuation appreciably increases the noise; moreover, for sufficiently low attenuations the fourth-order dispersion remains below the values of the second-order dispersion. Figure 1(b) shows the signal-to- noise ratios corresponding to the noise given in Fig. 1(a). It can be observed that the squeezed input improves the second- and fourth-order signal-to-noise ratios with respect to the coherent input. This improvement increases when the phases of the squeezed input are chosen to be $\theta=\arg \alpha=0$. On the other hand, the improvement also increases for lower attenuations, especially for the fourth-order ratio, which remains above the second-order ratio. Finally, it should be pointed out that the former results are in agreement with the analytical results given in Ref. 4.

If it is assumed that the attenuation effect of the optical fiber is equivalent to a two-photon absorption, the evolution of the signal-to-noise ratio along the transmission chain is that shown in Fig. 2. The attenuation of the fiber elements was taken to be slightly higher than the amplifier gain, making the noise of the coherent input rapidly equalize that of the squeezed input. Nevertheless, even in this case, the signal-to-noise ratios in second and fourth orders of the squeezed input remain above those
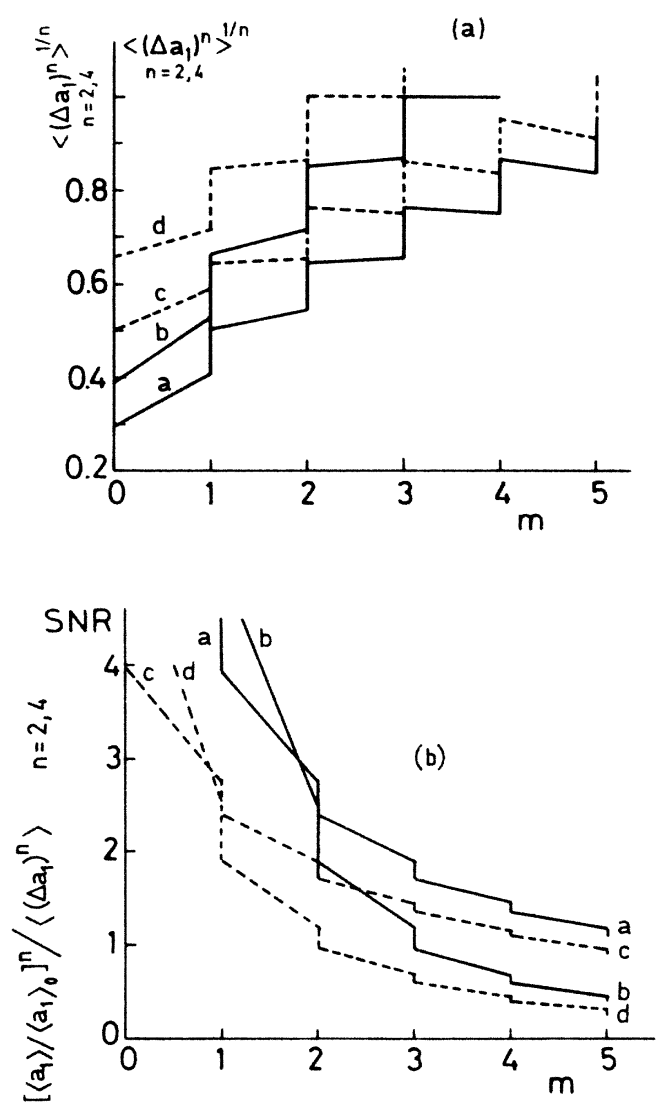

FIG. 1. Evolution of the noise and signal-to-noise ratio along a chain formed from linear elements for squeezed and coherent input states. (a) Evolution of noise for squeezed (curves $a$ and $b$ ) and coherent (curves $c$ and $d$ ) inputs. Curves $a$ and $c$ are second-order dispersions $\left\langle\left(\Delta a_{1}\right)^{2}\right\rangle^{1 / 2}$ and curves $b$ and $d$ are fourth-order dispersions $\left\langle\left(\Delta a_{1}\right)^{4}\right\rangle^{1 / 4}$. (b) Evolution of the corresponding signal-to-noise ratios $\left(\left\langle a_{1}\right\rangle /\left\langle a_{1}\right\rangle_{0}\right)^{2} /\left\langle\left(\Delta a_{1}\right)^{2}\right\rangle$, curves $a$ and $c$; and $\left(\left\langle a_{1}\right\rangle /\left\langle a_{1}\right\rangle_{0}\right)^{4} /\left\langle\left(\Delta a_{1}\right)^{4}\right\rangle$, curves $b$ and $d$, for the squeezed input (curves $a, b)$ and for the coherent input, characterized by $s=0$ (curves $c, d$ ). 


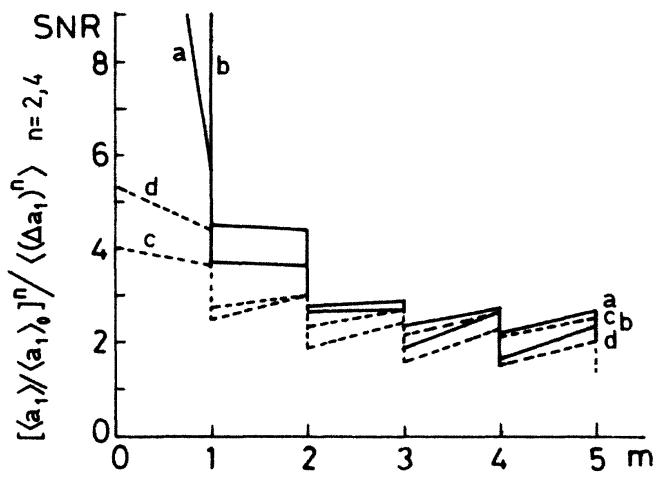

FIG. 2. Evolution of the signal-to-noise ratio in the case of alternating two-photon absorbers and linear amplifiers. The input state is characterized by a squeezing parameter $s=1$, phases $\theta=\arg \alpha=0$, and $\bar{n}_{0}=20$. The attenuation coefficient is $K=0.72$ and in the case of the coherent input $(s=0), K$ is slightly higher than the amplifier gain. The curves correspond to second-order (curves $a$ and $c$ ) and fourth-order (curves $b$ and $d$ ) signal-tonoise ratios for squeezed (curves $a$ and $b$ ) and coherent (curves $c$ and $d$ ) inputs.

corresponding to the coherent input. On the other hand, the signal-to-noise ratios $\left(\left\langle a_{1}\right\rangle /\left\langle a_{1}\right\rangle_{0}\right)^{2} /\left(\left(\Delta a_{1}\right)^{2}\right\rangle$ and $\left(\left\langle a_{1}\right\rangle /\left\langle a_{1}\right\rangle_{0}\right)^{4} /\left\langle\left(\Delta a_{1}\right)^{4}\right\rangle$ in Fig. 2 always remain appreciably above the signal-to-noise ratios when linear attenuators are assumed. Finally, it should be mentioned that if the transmission chain is assumed to be formed from alternating two-photon attenuators and two-photon amplifiers, the behavior of the signal-to-noise ratios is fairly degraded with respect to the case in which linear amplifiers are considered, both for squeezed and coherent input signals.

If we now assume that the attenuating elements of the transmission chain are three-photon absorbers, while the optical amplifiers are linear, the second- and fourth-order noises obtained are fairly lower than those obtained for two-photon absorbers. In the case of the coherent input, the effect of diminishing the input mean photon number is of decreasing the noise, but the corresponding signalto-noise ratios also decrease (Fig. 3). It is also shown in Fig. 3 that the signal-to-noise ratios remain constant

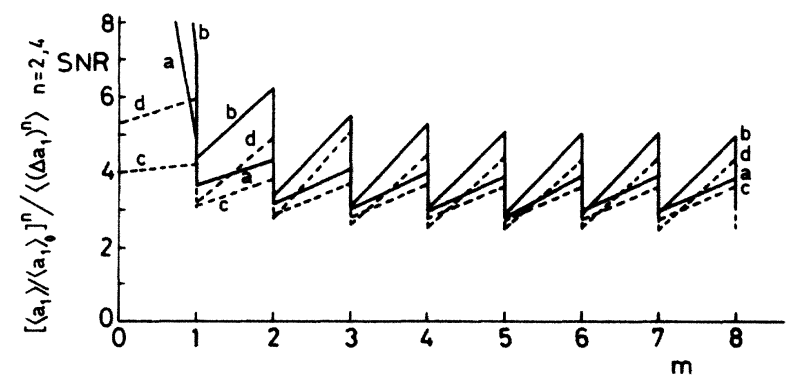

FIG. 3. Evolution of the signal-to-noise ratio along a chain formed from alternating three-photon absorbers and linear amplifiers. In the case of the coherent input, $K G=1$, but the input mean photon number is slightly lower than for the squeezed input $\left(\bar{n}_{0}=20\right)$. The other parameters are the same as in Fig. 2, and the curves are marked in the same way. from about the element of the chain $m=5$. Finally, it should be noted that for the conditions of Fig. 3, the fourth-order ratio remains above the second-order signal-to-noise ratio. If the attenuation of the chain elements is slightly lower than the amplifiers gain, the signal-to-noise ratio is appreciably improved with respect to the case in which $K \equiv G$, and conversely in the case in which $K<G^{-1}$. The behavior of the second- and fourth-order signal-to-noise ratios in a case where the attenuation is slightly smaller than the gain is depicted in Fig. 4. In such a case the signal-to-noise ratios $\left(\left\langle a_{1}\right\rangle /\left\langle a_{1}\right\rangle_{0}\right)^{2} /\left\langle\left(\Delta a_{1}\right)^{2}\right\rangle$ and $\left(\left\langle a_{1}\right\rangle /\left\langle a_{1}\right\rangle_{0}\right)^{4} /\left\langle\left(\Delta a_{1}\right)^{4}\right\rangle$ are appreciably improved with respect to the case in Fig. 3 in which $G=K$. The characteristics of both chains are very similar, since the slightly lower attenuation in Fig. 4 compared to that in Fig. 3 (which improves the signalto-noise ratios), is compensated by a lower-input mean photon number. On the other hand, the fourth-order signal-to-noise ratio again remains above the secondorder ratio. This behavior was not observed for low signal-to-noise ratios.

Finally, it is worth noting that when the transmission chain is formed from alternating three-photon absorbers and two-photon amplifiers, a highly ${ }^{15}$ squeezed input is needed to obtain signal-to-noise ratios similar to those corresponding to linear chains (Fig. 1).

In conclusion, the low-noise properties of squeezed light in a model of transmission link formed from alternating nonlinear attenuators and amplifiers have been evaluated. It was shown that when the attenuating sections of the fiber are assumed to be two-photon absorbers, the behavior of second- and fourth-order dispersions and signal-to-noise ratios is considerably improved with respect to the linear case. On the other hand, squeezed input light also improves the signal-to-noise ratios with respect to coherent inputs.

In the case of three-photon attenuators, we found that noise and signal-to-noise ratio remain practically constant from certain elements of the chain. Moreover, the fourth-order signal-to-noise ratios remain above secondorder ratios if sufficiently high signal-to-noise ratios are

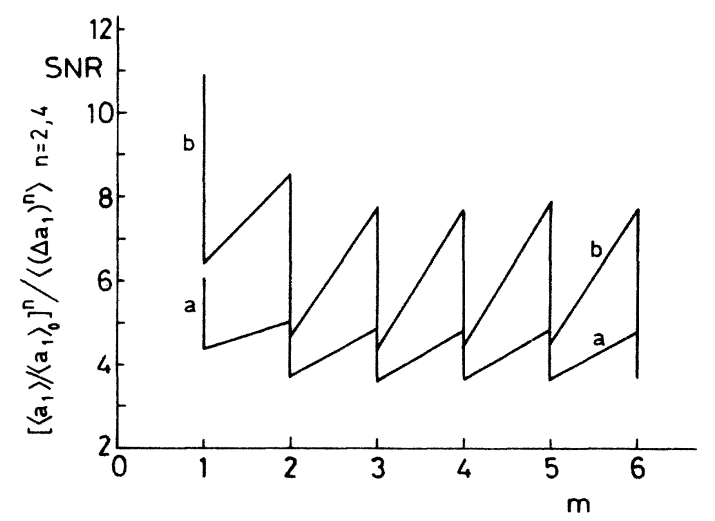

FIG. 4. Evolution of the second- (curve $a$ ) and fourth-order (curve $b$ ) signal-to-noise ratios along a chain formed from alternating three-photon absorbers and linear amplifiers. The attenuation $(K=0.8)$ is slightly lower than the amplifier gain, and the squeezed input is characterized by $s=1$ and $\bar{n}_{0}=16$. 
provided. It should be mentioned that when the fiber attenuation is slightly lower than the amplifier gain, the signal-to-noise ratios are appreciably improved. On the other hand we have shown that low attenuations and high-input mean photon numbers also improve the signal-to-noise ratios.

Finally, we have also shown that when the amplifiers are assumed to be nonlinear, the noise is increased and the signal-to-noise ratios considerably degraded, even when the input is highly squeezed. In summary, squeezed-input light and nonlinear absorbers appreciably improve the signal-to-noise ratios with respect to coherent inputs and linear chains. It should be stressed that we have dealt with an idealized model that does not take into account the deleterious effects due to nonlinearities, which can be very harmful in transmission schemes.
${ }^{1}$ R. Loudon, IEEE J. Quantum Electron. 21, 766 (1985).

${ }^{2}$ T. Mukai, Y. Yamamoto, and T. Kimura, IEEE J. Quantum Electron. 18, 1560 (1982).

${ }^{3}$ H. P. Yuen and J. H. Shapiro, IEEE Trans. Inf. Theory, 24, 657 (1978); J. H. Shapiro, H. P. Yuen, and J. A. Machado Mata, ibid. 25, 179 (1979); H. P. Yuen and J. H. Shapiro, ibid. 26, 78 (1980).

${ }^{4} \mathrm{R}$. Loudon, in Frontiers in Quantum Optics, Malvern Physics Series, edited by E. R. Pike and S. Sarkar (Hilger, Bristol, 1986), p. 42.

${ }^{5}$ R. E. Slusher, L. W. Hollberg, B. Yurke, J. C. Mertz, and J. F. Valley, Phys. Rev. Lett. 55, 2409 (1985); R. M. Shelby, M. D. Levenson, S. H. Perlmutter, R. G. DeVoe, and D. F. Walls, Phys. Rev. Lett. 57, 691 (1986).

${ }^{6}$ S. Carusotto, Opt. Acta 27, 1567 (1980).

${ }^{7}$ M. S. Zubairy and J. J. Yeh, Phys. Rev. A 21, 1624 (1980).

${ }^{8}$ L. Sainz and P. F. González-Díaz, Phys. Rev. A 31, 1598 (1985).
${ }^{9}$ F. J. Bermejo and L. Sainz, Comput. Phys. Commun. 43, 245 (1987); P. García-Fernández and F. J. Bermejo, Phys. Lett. 118A, 400 (1986); L. Sainz and F. J. Bermejo, ibid. 114A, 121 (1986).

${ }^{10}$ H. P. Yuen, Phys. Rev. A 13, 2226 (1976); C. M. Caves, Phys. Rev. D 23, 1693 (1981).

${ }^{11}$ C. K. Hong and L. Mandel, Phys. Rev. A 32, 974 (1985); Phys. Rev. Lett. 54, 323 (1985).

${ }^{12}$ S. Friberg and L. Mandel, Opt. Commun. 46, 141 (1983).

${ }^{13}$ R. Loudon and T. J. Shepherd, Opt. Acta 31, 1243 (1984).

${ }^{14}$ P. García-Fernández, F. J. Bermejo, and L. Sainz, J. Mod. Opt. 34, 997 (1987); P. García-Fernández and F. J. Bermejo (unpublished).

${ }^{15}$ In the case of highly squeezed inputs $(s=1.4)$, we found the same oscillations in the photon distributions as those recently analyzed by W. Schleich and J. A. Wheeler, Nature 326, 574 (1987). 